\title{
A Remark on the Regularity Criterion for the MHD Equations via Two Components in Morrey-Campanato Spaces
}

\author{
Zujin Zhang, ${ }^{1}$ Tong Tang, ${ }^{2}$ and Fumin Zhang' \\ ${ }^{1}$ School of Mathematics and Computer Sciences, Gannan Normal University, Ganzhou, Jiangxi 341000, China \\ ${ }^{2}$ Department of Mathematics, College of Sciences, Hohai University, Nanjing 210098, China
}

Correspondence should be addressed to Zujin Zhang; zhangzujin361@163.com

Received 1 April 2014; Accepted 9 June 2014; Published 25 June 2014

Academic Editor: Richard Saurel

Copyright (c) 2014 Zujin Zhang et al. This is an open access article distributed under the Creative Commons Attribution License, which permits unrestricted use, distribution, and reproduction in any medium, provided the original work is properly cited.

We consider the regularity criterion for the 3D MHD equations. It is proved that if the horizontal components of the velocity and magnetic fields satisfy $\widetilde{\mathbf{u}}, \widetilde{\mathbf{b}} \in L^{2 /(1-r)}\left(0, T ; \dot{M}_{2,3 / r}\right)$ with $0<r<1$, then the solution smooth. This improves the result given by Gala (2012).

\section{Introduction}

In this paper, we consider the following three-dimensional (3D) magnetohydrodynamic (MHD) equations:

$$
\begin{gathered}
\mathbf{u}_{t}+(\mathbf{u} \cdot \nabla) \mathbf{u}-(\mathbf{b} \cdot \nabla) \mathbf{b}-\Delta \mathbf{u}+\nabla \pi=\mathbf{0}, \\
\mathbf{b}_{t}+(\mathbf{u} \cdot \nabla) \mathbf{b}-(\mathbf{b} \cdot \nabla) \mathbf{u}-\Delta \mathbf{b}=\mathbf{0}, \\
\nabla \cdot \mathbf{u}=0, \\
\nabla \cdot \mathbf{b}=0, \\
\mathbf{u}(0)=\mathbf{u}_{0}, \quad \mathbf{b}(0)=\mathbf{b}_{0},
\end{gathered}
$$

where $\mathbf{u}=\left(u_{1}, u_{2}, u_{3}\right)$ is the fluid velocity field, $\mathbf{b}=$ $\left(b_{1}, b_{2}, b_{3}\right)$ is the magnetic field, $\pi$ is a scalar pressure, and $\left(\mathbf{u}_{0}, \mathbf{b}_{0}\right)$ are the prescribed initial data satisfying $\nabla \cdot \mathbf{u}_{0}=$ $\nabla \cdot \mathbf{b}_{0}=0$ in the distributional sense. Physically, (1) governs the dynamics of the velocity and magnetic fields in electrically conducting fluids, such as plasmas, liquid metals, and salt water. Moreover, $(1)_{1}$ reflects the conservation of momentum, $(1)_{2}$ is the induction equation, and $(1)_{3}$ specifies the conservation of mass.

Besides its physical applications, the MHD system (1) is also mathematically significant. Duvaut and Lions [1] constructed a global weak solution to (1) for initial data with finite energy. However, the issue of regularity and uniqueness of such a given weak solution remains a challenging open problem. Many sufficient conditions (see, e.g., [2-14] and the references therein) were derived to guarantee the regularity of the weak solution. Among these results, we are interested in regularity criteria involving only partial components of the velocity $\mathbf{u}$, the magnetic field $\mathbf{b}$, the pressure gradient $\nabla \pi$, and so forth.

$\mathrm{CaO}$ and $\mathrm{Wu}[2]$ proved the following regularity criterion:

$$
\partial_{3} \mathbf{u} \in L^{p}\left(0, T ; L^{q}\left(\mathbb{R}^{3}\right)\right), \quad \frac{2}{p}+\frac{3}{q}=1, \quad 3 \leq q \leq \infty .
$$

Jia and Zhou showed that if

$$
\partial_{3} \mathbf{u} \in L^{p}\left(0, T ; L^{q}\left(\mathbb{R}^{3}\right)\right), \quad \frac{2}{p}+\frac{3}{q}=\frac{3}{4}+\frac{2}{2 q}, \quad q>2,
$$

then the solution is regular. These results were improved by Zhang [15] to be

$$
\partial_{3} \mathbf{u} \in L^{p}\left(0, T ; L^{q}\left(\mathbb{R}^{3}\right)\right), \quad \frac{2}{p}+\frac{3}{q}=1+\frac{1}{q}, \quad q>2 .
$$

Once only partial components of the velocity field are concerned, we have combinatoric regularity criterion involving partial components of the magnetic field also. This is due partially to the strong coupling of the velocity and magnetic fields. Let us list some recent progress. Gala and 
Lemarié-Rieusset [6] established the following two regularity conditions:

$$
\begin{array}{rr}
\left(u_{1}, u_{2}\right) \in L^{p}\left(0, T ; L^{q}\left(\mathbb{R}^{3}\right)\right) \quad \text { with } \frac{2}{p}+\frac{3}{q}=1, \\
3<q \leq \infty, \\
\left(b_{1}, b_{2}\right) \in L^{r}\left(0, T ; L^{s}\left(\mathbb{R}^{3}\right)\right), \quad \text { with } \frac{2}{r}+\frac{3}{s}=1, \\
3<s \leq \infty, \\
\left(u_{1}, u_{2}\right) \in L^{p}\left(0, T ; L^{q}\left(\mathbb{R}^{3}\right)\right), \quad b_{3} \in L^{r}\left(0, T ; L^{s}\left(\mathbb{R}^{3}\right)\right), \\
\text { with }\left(\frac{2}{p}+\frac{3}{q}\right)+\left(\frac{2}{r}+\frac{3}{s}\right) \leq 2, \quad \frac{2}{p}+\frac{3}{q} \leq 1,3<q \leq \infty .
\end{array}
$$

Recently, Ni et al. in [10] showed that each of the following three conditions

$$
\begin{gathered}
\nabla_{h} \mathbf{u} \in L^{p}\left(0, T ; L^{q}\left(\mathbb{R}^{3}\right)\right), \quad \text { with } \frac{2}{p}+\frac{3}{q}=2, \frac{3}{2}<q \leq \infty, \\
\partial_{3} \mathbf{b} \in L^{r}\left(0, T ; L^{s}\left(\mathbb{R}^{3}\right)\right), \quad \text { with } \frac{2}{r}+\frac{3}{s}=2, \frac{3}{2}<s \leq \infty ;
\end{gathered}
$$

or

$$
\begin{aligned}
& \nabla_{h} \mathbf{u} \in L^{p}\left(0, T ; L^{q}\left(\mathbb{R}^{3}\right)\right), \quad \text { with } \frac{2}{p}+\frac{3}{q}=2, \frac{3}{2}<q \leq \infty, \\
& \nabla_{h} \mathbf{b} \in L^{r}\left(0, T ; L^{s}\left(\mathbb{R}^{3}\right)\right), \quad \text { with } \frac{2}{r}+\frac{3}{s}=2, \frac{3}{2}<s \leq \infty ;
\end{aligned}
$$

or

$$
\begin{gathered}
u_{3} \in L^{p_{1}}\left(0, T ; L^{q_{1}}\left(\mathbb{R}^{3}\right)\right), \quad \text { with } \frac{2}{p_{1}}+\frac{3}{q_{1}}=1,3<q \leq \infty, \\
\partial_{3} \mathbf{u} \in L^{p_{2}}\left(0, T ; L^{q_{2}}\left(\mathbb{R}^{3}\right)\right), \quad \text { with } \frac{2}{p_{2}}+\frac{3}{q_{2}}=2, \\
\frac{3}{2}<q \leq \infty,
\end{gathered}
$$

$\mathbf{b} \in L^{p_{3}}\left(0, T ; L^{q_{3}}\left(\mathbb{R}^{3}\right)\right)$, with $\frac{2}{p_{3}}+\frac{3}{q_{3}}=1,3<q \leq \infty$,

$$
\begin{aligned}
& \partial_{3} \mathbf{b} \in L^{p_{4}}\left(0, T ; L^{q_{4}}\left(\mathbb{R}^{3}\right)\right), \quad \text { with } \frac{2}{p_{4}}+\frac{3}{q_{4}}=2, \\
& \frac{3}{2}<q \leq \infty,
\end{aligned}
$$

ensures the smoothness of the solution. Here, $\nabla_{h}=\left(\partial_{1}, \partial_{2}\right)$ is the horizontal gradient operator.

The motivation of this paper is to give another contribution in this direction. Motivated by [8], we would like to show the following regularity condition for (1):

$$
\widetilde{\mathbf{u}}, \widetilde{\mathbf{b}} \in L^{2 /(1-r)}\left(0, T ; \dot{M}_{2,3 / r}\right) \quad \text { with } 0<r<1 .
$$

Here, and in what follows, we denote by

$$
\widetilde{\mathbf{u}}=\left(u_{1}, u_{2}, 0\right), \quad \widetilde{\mathbf{b}}=\left(b_{1}, b_{2}, 0\right)
$$

the horizontal components of the velocity and magnetic fields, respectively, and by

$$
\widetilde{\nabla}=\left(\partial_{1}, \partial_{2}, 0\right) \quad \text { with } \partial_{i}=\frac{\partial}{\partial x_{i}}
$$

the horizontal gradient operator.

Before stating the precise result, let us recall the weak formulation of the MHD equations (1).

Definition 1. Let $\left(\mathbf{u}_{0}, \mathbf{b}_{0}\right) \in L^{2}\left(\mathbb{R}^{3}\right)$ with $\nabla \cdot \mathbf{u}_{0}=\nabla \cdot \mathbf{b}_{0}=0$ and $T>0$. A measurable $\mathbb{R}^{3}$-valued pair $(\mathbf{u}, \mathbf{b})$ is called a weak solution to (1) with initial data $\left(\mathbf{u}_{0}, \mathbf{b}_{0}\right)$, provided that the following three conditions hold:

(1) $\mathbf{u} \in L^{\infty}\left(0, T ; L^{2}\left(\mathbb{R}^{3}\right)\right) \cap L^{2}\left(0, T ; H^{1}\left(\mathbb{R}^{3}\right)\right)$, b $\in$ $L^{\infty}\left(0, T ; L^{2}\left(\mathbb{R}^{3}\right)\right) \cap L^{2}\left(0, T ; H^{1}\left(\mathbb{R}^{3}\right)\right) ;$

(2) $(1)_{1,2,3,4}$ are satisfied in the distributional sense;

(3) the energy inequality

$$
\|(\mathbf{u}, \mathbf{b})\|_{L^{2}}^{2}(t)+2 \int_{0}^{t}\|\nabla(\mathbf{u}, \mathbf{b})\|_{L^{2}}^{2}(s) \mathrm{d} s \leq\left\|\left(\mathbf{u}_{0}, \mathbf{b}_{0}\right)\right\|_{L^{2}}^{2} .
$$

Now, our main result reads as follows.

Theorem 2. Let $\left(\mathbf{u}_{0}, \mathbf{b}_{0}\right) \in H^{1}\left(\mathbb{R}^{3}\right)$ with $\nabla \cdot \mathbf{u}_{0}=\nabla \cdot \mathbf{b}_{0}=0$ and $T>0$. Assume that $(\mathbf{u}, \mathbf{b})$ is a given weak solution pair of the MHD system (1) with initial data $\left(\mathbf{u}_{0}, \mathbf{b}_{0}\right)$ on $(0, T)$. If

$$
\widetilde{\mathbf{u}}, \widetilde{\mathbf{b}} \in L^{2 /(1-r)}\left(0, T ; \dot{M}_{2,3 / r}\right) \quad \text { with } 0<r<1 \text {, }
$$

then the solution is smooth on $(0, T)$.

Here, $\dot{M}_{p, q}$ is the Morrey-Campanato space, which will be introduced in Section 2. And Section 3 is devoted to the proof of Theorem 2 .

Remark 3. Noticing that $\dot{X}_{r} \subset \dot{M}_{2,3 / r}$ for $0<r<1$ (see (19)), we indeed improve the result of [16].

\section{Preliminaries}

In this section, we will introduce the definition of MorreyCampanato space $\dot{M}_{p, q}$ and recall its fundamental properties. The space plays an important role in studying the regularity of solutions to partial differential equations (see $[11,17,18]$, e.g.).

Definition 4. For $1<p \leq q \leq+\infty$, the Morrey-Campanato space $\dot{M}_{p, q}$ is defined as

$$
\begin{aligned}
\dot{M}_{p, q}=\{f & \in L_{\text {loc }}^{p}\left(\mathbb{R}^{3}\right) ;\|f\|_{\dot{M}_{p, q}}=\sup _{x \in \mathbb{R}^{3}, R>0} \frac{1}{R^{(3 / p)-(3 / q)}} \\
& \left.\times\left(\int_{B(x, R)}|f(y)|^{p} \mathrm{~d} y\right)^{1 / p}<+\infty\right\},
\end{aligned}
$$

where $B(x, R) \subset \mathbb{R}^{3}$ is the ball with center $x$ and radius $R$. 
One sees readily that $\dot{M}_{p, q}$ is a Banach space under the norm $\|\cdot\|_{\dot{M}_{p, q}}$ and contains the classical Lebesgue space as a subspace:

$$
L^{q}=\dot{M}_{q, q} \subset \dot{M}_{p, q} .
$$

Moreover, the following scaling property holds:

$$
\|f(\lambda \cdot)\|_{\dot{M}_{p, q}}=\frac{1}{\lambda^{(3 / q)}}\|f\|_{\dot{M}_{p, q}}, \quad \text { for } \lambda>0 .
$$

Due to the following characterization in [19].

Lemma 5. For $0 \leq r<3 / 2$, the space $\dot{Z}_{r}$ is defined as the space of all functions $f \in L_{\mathrm{loc}}^{2}\left(\mathbb{R}^{3}\right)$ such that

$$
\|f\|_{\dot{Z}_{r}}=\sup _{\|g\|_{\dot{2}_{2,1}^{r}} \leq 1}\|f g\|_{L^{2}}<+\infty .
$$

Then, $f \in \dot{M}_{2,3 / r}$ if and only if $f \in \dot{Z}_{r}$ with equivalent norm.

And, with the fact that

$$
L^{2} \cap \dot{H}^{r} \subset \dot{B}_{2,1}^{r} \subset \dot{H}^{r} \quad \text { for } 0<r<1,
$$

we have

$$
\dot{X}_{r} \subset \dot{M}_{2,3 / r} \text {. }
$$

Here, $\dot{B}_{2,1}^{r}$ is the Besov space, which is intermediate between $L^{2}$ and $\dot{H}^{1}$ (see [20]):

$$
\|f\|_{\dot{B}_{2,1}^{r}} \leq C\|f\|_{L^{2}}^{1-r}\|\nabla f\|_{L^{2}}^{r}, \quad \text { for } 0<r<1 .
$$

\section{Proof of Theorem 2}

In this section, we will prove Theorem 2 .

It is well known (see [21], e.g.) that, for $\mathbf{u}_{0} \in H^{1}\left(\mathbb{R}^{3}\right)$ with $\nabla \cdot \mathbf{u}_{0}=0$, (1) possesses a local strong solution

$$
(\mathbf{u}, \mathbf{b}) \in L^{\infty}\left(0, \Gamma^{*} ; H^{1}\left(\mathbb{R}^{3}\right)\right) \cap L^{2}\left(0, \Gamma^{*} ; H^{2}\left(\mathbb{R}^{3}\right)\right),
$$

where $\Gamma^{*}$ is the maximal existence of the strong solution. Moreover, this strong solution is smooth and unique in the class of weak solutions. Thus, if $\Gamma^{*} \geq T$, we have nothing to prove. Otherwise, we will show that the $H^{1}$ norm of this strong solution remains bounded as $t \in\left[0, \Gamma^{*}\right)$. The standard continuation argument then yields that $\Gamma^{*}$ could not be the maximal existence of the strong solution. This contradiction concludes that $\Gamma^{*} \geq T$, and we complete the proof.

Taking the inner product of $(1)_{1}$ with $-\Delta \mathbf{u},(1)_{2}$ with $-\Delta \mathbf{b}$ in $L^{2}\left(\mathbb{R}^{3}\right)$, respectively, and adding the resulting equations together, we obtain

$$
\begin{aligned}
\frac{1}{2} \frac{\mathrm{d}}{\mathrm{d} t} & \|\nabla(\mathbf{u}, \mathbf{b})\|_{L^{2}}^{2}+\|\Delta(\mathbf{u}, \mathbf{b})\|_{L^{2}}^{2} \\
= & \int_{\mathbb{R}^{3}}[(\mathbf{u} \cdot \nabla) \mathbf{u}] \cdot \Delta \mathbf{u} \mathrm{d} x-\int_{\mathbb{R}^{3}}[(\mathbf{b} \cdot \nabla) \mathbf{u}] \cdot \Delta \mathbf{u} \mathrm{d} x \\
& \quad+\int_{\mathbb{R}^{3}}[(\mathbf{u} \cdot \nabla) \mathbf{b}] \cdot \Delta \mathbf{b} \mathrm{d} x-\int_{\mathbb{R}^{3}}[(\mathbf{b} \cdot \nabla) \mathbf{u}] \cdot \Delta \mathbf{b} \mathrm{d} x
\end{aligned}
$$

$$
\begin{aligned}
= & -\sum_{k=1}^{3} \int_{\mathbb{R}^{3}}\left[\left(\partial_{k} \mathbf{u} \cdot \nabla\right) \mathbf{u}\right] \cdot \partial_{k} \mathbf{u} \mathrm{d} x \\
& +\sum_{k=1}^{3} \int_{\mathbb{R}^{3}}\left[\left(\partial_{k} \mathbf{b} \cdot \nabla\right) \mathbf{b}\right] \cdot \partial_{k} \mathbf{u} \mathrm{d} x \\
& -\sum_{k=1}^{3} \int_{\mathbb{R}^{3}}\left[\left(\partial_{k} \mathbf{u} \cdot \nabla\right) \mathbf{b}\right] \cdot \partial_{k} \mathbf{b} \mathrm{d} x \\
& +\sum_{k=1}^{3} \int_{\mathbb{R}^{3}}\left[\left(\partial_{k} \mathbf{b} \cdot \nabla\right) \mathbf{u}\right] \cdot \partial_{k} \mathbf{b} \mathrm{d} x \\
\equiv & I_{1}+I_{2}+I_{3}+I_{4},
\end{aligned}
$$

where we use integration by parts formula, the fact that

$$
\nabla \cdot \mathbf{u}=\nabla \cdot \mathbf{b}=0
$$

and its consequence

$$
\int_{\mathbb{R}^{3}}\left[(\mathbf{b} \cdot \nabla) \partial_{k} \mathbf{b}\right] \cdot \partial_{k} \mathbf{u}+\left[(\mathbf{b} \cdot \nabla) \partial_{k} \mathbf{u}\right] \cdot \partial_{k} \mathbf{b} \mathrm{d} x=0 .
$$

For $I_{1}$,

$$
\begin{aligned}
I_{1}= & -\sum_{k=1}^{3} \int_{\mathbb{R}^{3}}\left[\left(\partial_{k} \widetilde{\mathbf{u}} \cdot \widetilde{\nabla}\right) \mathbf{u}\right] \cdot \partial_{k} \mathbf{u} \mathrm{d} x \\
& -\sum_{k=1}^{3} \int_{\mathbb{R}^{3}} \partial_{k} u_{3} \partial_{3} \widetilde{\mathbf{u}} \cdot \partial_{k} \widetilde{\mathbf{u}} \mathrm{d} x \\
& -\sum_{k=1}^{3} \int_{\mathbb{R}^{3}} \partial_{k} u_{3} \partial_{3} u_{3} \partial_{k} u_{3} \mathrm{~d} x .
\end{aligned}
$$

Integrating by parts and noticing that $\partial_{3} u_{3}=-\partial_{1} u_{1}-\partial_{2} u_{2}$, we get

$$
I_{1} \leq C \int_{\mathbb{R}^{3}}|\widetilde{\mathbf{u}}| \cdot|\nabla \mathbf{u}| \cdot\left|\nabla^{2} \mathbf{u}\right| \mathrm{d} x .
$$

The remaining terms $I_{2}, I_{3}, I_{4}$ can be similarly decomposed and bounded so that

$$
I_{2}, I_{3}, I_{4} \leq C \int_{\mathbb{R}^{3}}|(\widetilde{\mathbf{u}}, \widetilde{\mathbf{b}})| \cdot|\nabla(\mathbf{u}, \mathbf{b})| \cdot\left|\nabla^{2}(\mathbf{u}, \mathbf{b})\right| \mathrm{d} x .
$$

Plugging (26) and (27) into (22), we gather

$$
\begin{aligned}
& \frac{1}{2} \frac{\mathrm{d}}{\mathrm{d} t}\|\nabla(\mathbf{u}, \mathbf{b})\|_{L^{2}}^{2}+\|\Delta(\mathbf{u}, \mathbf{b})\|_{L^{2}}^{2} \\
& \leq C \int_{\mathbb{R}^{3}}|(\widetilde{\mathbf{u}}, \widetilde{\mathbf{b}})| \cdot|\nabla(\mathbf{u}, \mathbf{b})| \cdot\left|\nabla^{2}(\mathbf{u}, \mathbf{b})\right| \mathrm{d} x \\
& \leq C\||(\widetilde{\mathbf{u}}, \widetilde{\mathbf{b}})| \cdot|\nabla(\mathbf{u}, \mathbf{b})|\|_{L^{2}}\left\|\nabla^{2}(\mathbf{u}, \mathbf{b})\right\|_{L^{2}} \\
& \leq C\|(\widetilde{\mathbf{u}}, \widetilde{\mathbf{b}})\|_{\dot{M}_{2,3 / r}}\|\nabla(\mathbf{u}, \mathbf{b})\|_{\dot{B}_{2,1}^{r}}\|\Delta(\mathbf{u}, \mathbf{b})\|_{L^{2}} \text { (by Lemma 5) } \\
& \left.\leq C\|(\widetilde{\mathbf{u}}, \widetilde{\mathbf{b}})\|_{\dot{M}_{2,3 / r}}\|\nabla(\mathbf{u}, \mathbf{b})\|_{L^{2}}^{1-r}\|\Delta(\mathbf{u}, \mathbf{b})\|_{L^{2}}^{1+r} \text { (by }(20)\right) \\
& \leq C\|(\widetilde{\mathbf{u}}, \widetilde{\mathbf{b}})\|_{\dot{M}_{2,3 / r}}^{2 /(1-r)}\|\nabla(\mathbf{u}, \mathbf{b})\|_{L^{2}}^{2}+\frac{1}{2}\|\Delta(\mathbf{u}, \mathbf{b})\|_{L^{2}}^{2} .
\end{aligned}
$$


Consequently,

$$
\frac{\mathrm{d}}{\mathrm{d} t}\|\nabla(\mathbf{u}, \mathbf{b})\|_{L^{2}}^{2}+\|\Delta(\mathbf{u}, \mathbf{b})\|_{L^{2}}^{2} \leq C\|(\widetilde{\mathbf{u}}, \widetilde{\mathbf{b}})\|_{\dot{M}_{2,3 / r}}^{2 /(1-r)}\|\nabla(\mathbf{u}, \mathbf{b})\|_{L^{2}}^{2}
$$

Applying Gronwall inequality, we deduce that

$$
\begin{aligned}
& \|\nabla(\mathbf{u}, \mathbf{b})\|_{L^{2}}^{2}(t) \\
& \quad \leq\left\|\nabla\left(\mathbf{u}_{0}, \mathbf{b}_{0}\right)\right\|_{L^{2}}^{2} \cdot \exp \left\{C \int_{0}^{T}\|(\widetilde{\mathbf{u}}, \widetilde{\mathbf{b}})\|_{\dot{M}_{2,3 / r}}^{2 /(1-r)}(s) \mathrm{d} s\right\}<+\infty .
\end{aligned}
$$

As soon as the estimates of $\|\nabla(\mathbf{u}, \mathbf{b})\|_{L^{2}}$ are obtained, we can invoke the standard energy method to bootstrap the solution to be in $W^{k, 2}\left(\mathbb{R}^{3}\right)$ for all $k \in \mathbb{Z}^{+}$. The Sobolev imbedding theorem then implies that the solution is smooth.

The proof of Theorem 2 is completed.

\section{Conflict of Interests}

The authors declare that there is no conflict of interests regarding the publication of this paper.

\section{Acknowledgments}

This work was partially supported by the National Natural Science Foundation of China (11326138), the Youth Natural Science Foundation of Jiangxi Province (20132BAB211007), and the Science Foundation of Jiangxi Provincial Department of Education (GJJ14673).

\section{References}

[1] G. Duvaut and J. Lions, "Inéquations en thermoélasticité et magnétohydrodynamique," Archive for Rational Mechanics and Analysis, vol. 46, pp. 241-279, 1972.

[2] C. Cao and J. H. Wu, "Two regularity criteria for the 3D MHD equations," Journal of Differential Equations, vol. 248, no. 9, pp. 2263-2274, 2010.

[3] Q. Chen, C. Miao, and Z. Zhang, "On the regularity criterion of weak solution for the 3D viscous magneto-hydrodynamics equations," Communications in Mathematical Physics, vol. 284, no. 3, pp. 919-930, 2008.

[4] H. L. Duan, "On regularity criteria in terms of pressure for the $3 \mathrm{D}$ viscous MHD equations," Applicable Analysis, vol. 91, no. 5, pp. 947-952, 2012.

[5] C. He and Z. Xin, "On the regularity of weak solutions to the magnetohydrodynamic equations," Journal of Differential Equations, vol. 213, no. 2, pp. 235-254, 2005.

[6] S. Gala and P. G. Lemarié-Rieusset, "Some regularity criteria for the 3D incompressible magnetohydrodynamics," Journal of Mathematical Analysis and Applications, vol. 369, no. 1, pp. 317$322,2010$.

[7] X. J. Jia and Y. Zhou, "A new regularity criterion for the 3D incompressible MHD equations in terms of one component of the gradient of pressure," Journal of Mathematical Analysis and Applications, vol. 396, no. 1, pp. 345-350, 2012.
[8] X. Jia and Y. Zhou, "Regularity criteria for the 3D MHD equations involving partial components," Nonlinear Analysis. Real World Applications. An International Multidisciplinary Journal, vol. 13, no. 1, pp. 410-418, 2012.

[9] X. Jia and Y. Zhou, "Regularity criteria for the 3D MHD equations via partial derivatives," Kinetic and Related Models, vol. 5, no. 3, pp. 505-516, 2012.

[10] L. D. Ni, Z. G. Guo, and Y. Zhou, "Some new regularity criteria for the 3D MHD equations," Journal of Mathematical Analysis and Applications, vol. 396, no. 1, pp. 108-118, 2012.

[11] Z. Zhang, "Remarks on the regularity criteria for generalized MHD equations," Journal of Mathematical Analysis and Applications, vol. 375, no. 2, pp. 799-802, 2011.

[12] Z. Zhang, P. Li, and G. Yu, "Regularity criteria for the 3D MHD equations via one directional derivative of the pressure," Journal of Mathematical Analysis and Applications, vol. 401, no. 1, pp. 66-71, 2013.

[13] Y. Zhou, "Remarks on regularities for the 3D MHD equations," Discrete and Continuous Dynamical Systems A, vol. 12, no. 5, pp. 881-886, 2005.

[14] Y. Zhou and J. Fan, "Logarithmically improved regular ity criteria for the 3D viscous MHD equations," Forum Mathematicum, vol. 24, no. 4, pp. 691-708, 2012.

[15] Z. J. Zhang, "Mageto-micropolar uid equations with regularity in one direction".

[16] S. Gala, "A new regularity criterion for the 3D MHD equations in $\mathrm{R}_{3}$," Communications on Pure and Applied Analysis, vol. 11, pp. 937-980, 2012.

[17] T. Kato, "Strong solutions of the Navier-Stokes equation in Morrey spaces," Boletim da Sociedade Brasileira de Matemática, vol. 22, no. 2, pp. 127-155, 1992.

[18] M. E. Taylor, "Analysis on Morrey spaces and applications to Navier-Stokes and other evolution equations," Communications in Partial Differential Equations, vol. 17, no. 9-10, pp. 1407-1456, 1992.

[19] P. G. Lemarié-Rieusset, Recent Developments in the NavierStokes Problem, Chapman and Hall, London, UK, 2002.

[20] Y. Zhou and S. Gala, "A new regularity criterion for weak solutions to the viscous MHD equations in terms of the vorticity field," Nonlinear Analysis: Theory, Methods \& Applications, vol. 72, no. 9-10, pp. 3643-3648, 2010.

[21] M. Sermange and R. Temam, "Some mathematical questions related to the MHD equations," Communications on Pure and Applied Mathematics, vol. 36, no. 5, pp. 635-664, 1983. 


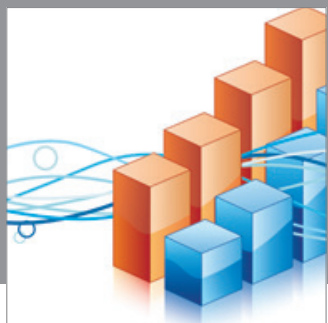

Advances in

Operations Research

mansans

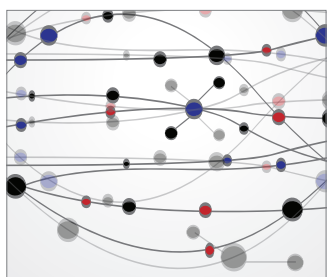

The Scientific World Journal
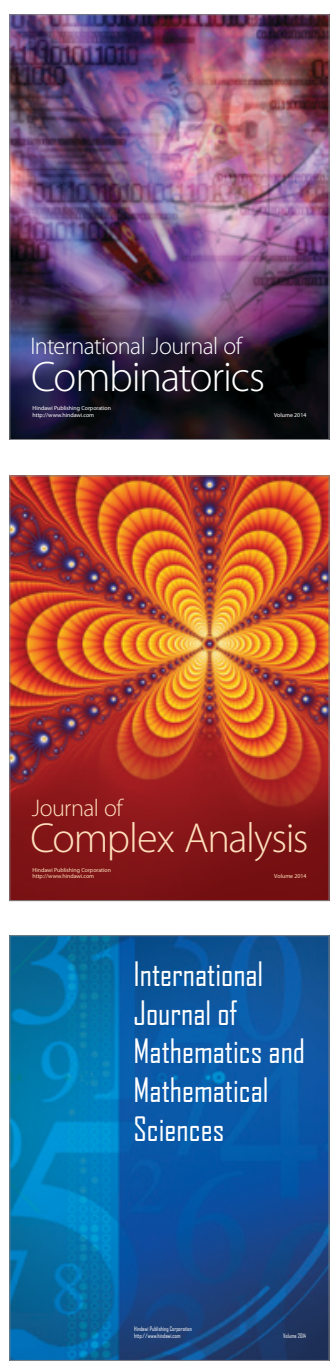
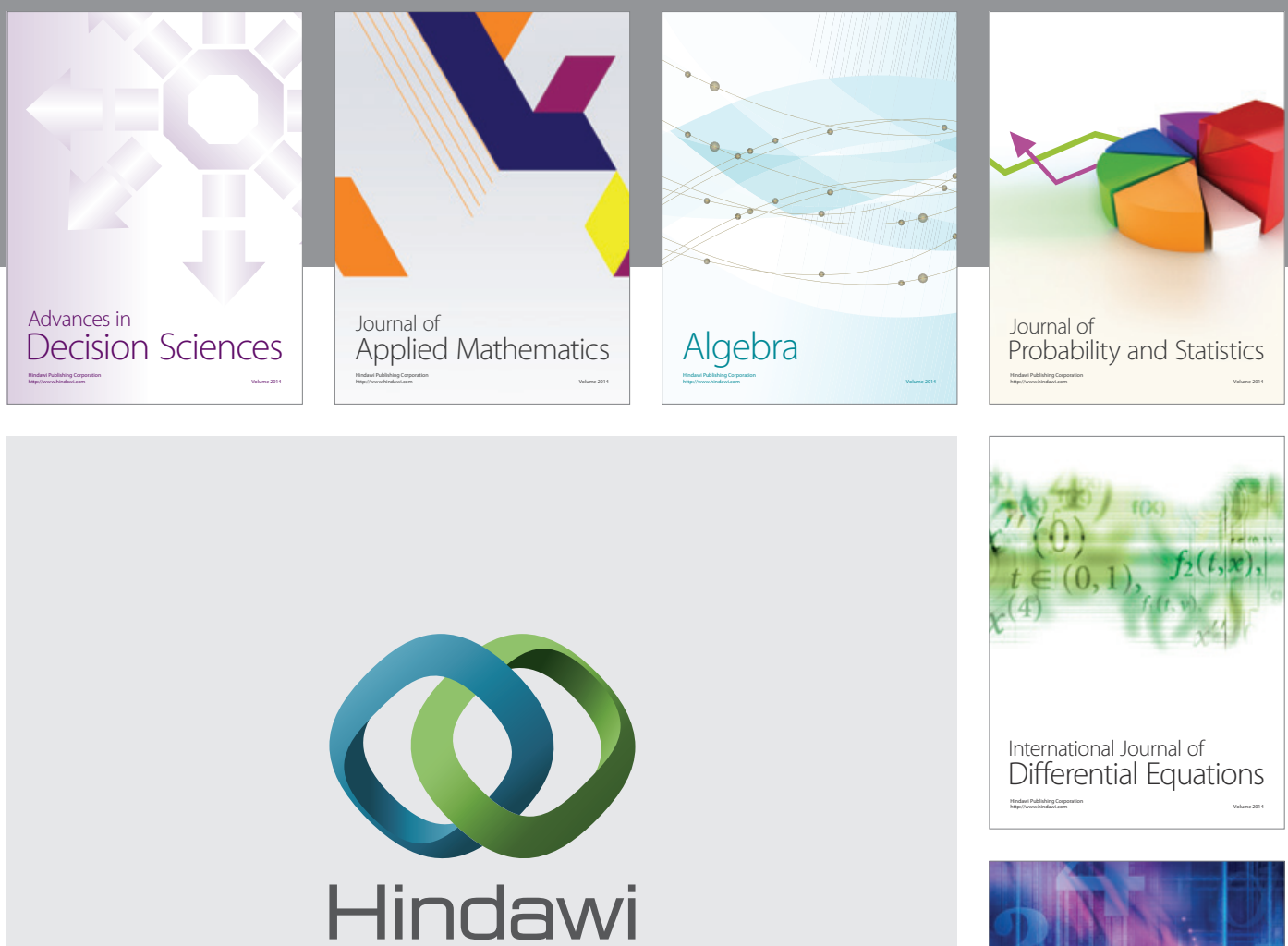

Submit your manuscripts at http://www.hindawi.com
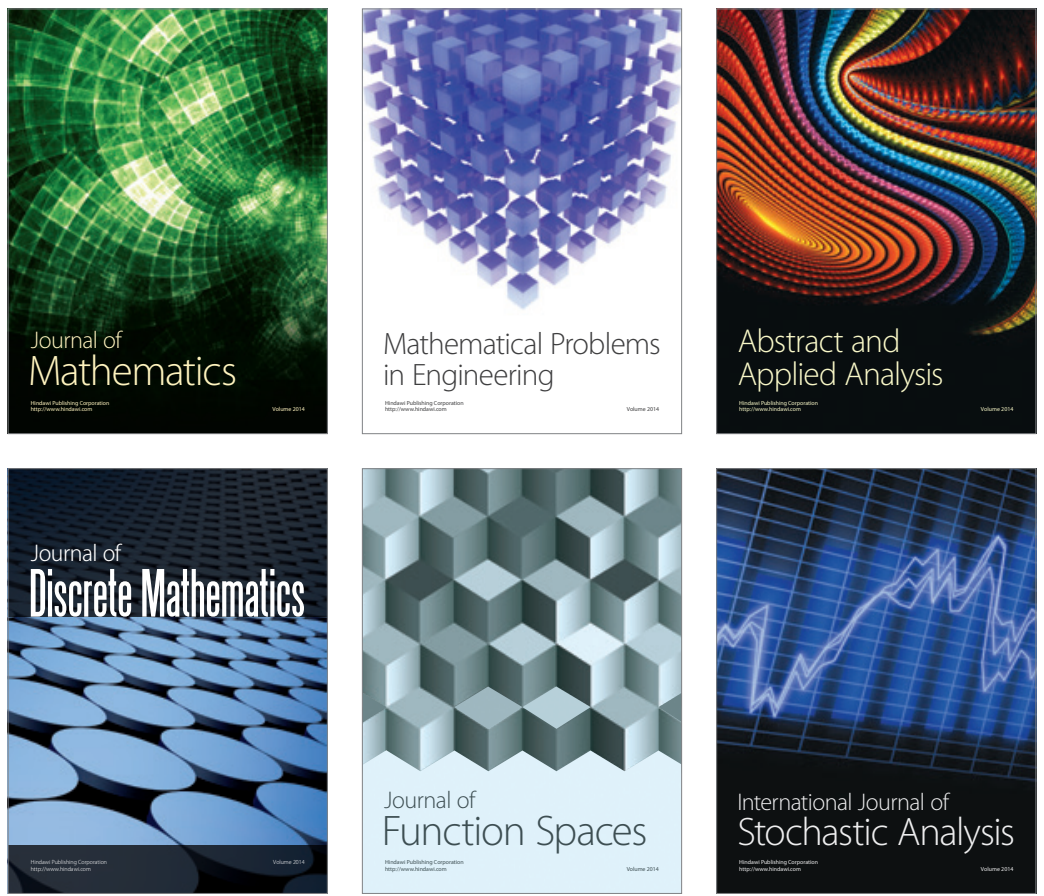

Journal of

Function Spaces

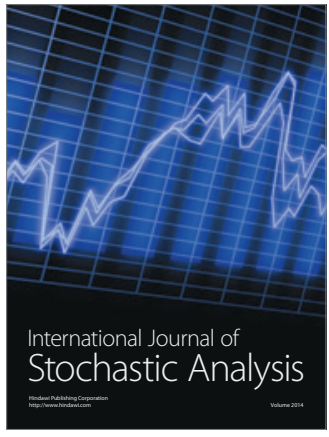

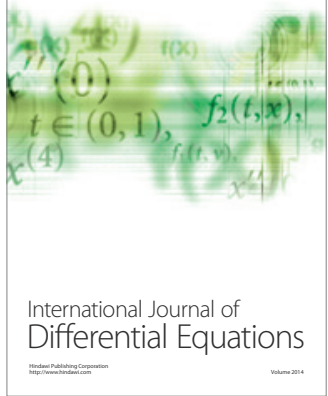
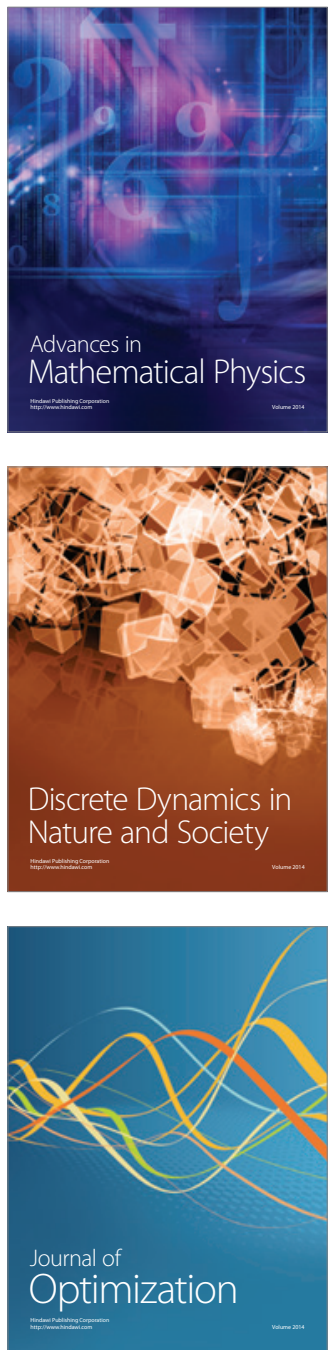